\title{
Solving local anomaly equations in gauge-rank extensions of the standard model
}

\author{
B. C. Allanach $\oplus^{*}$ \\ DAMTP, University of Cambridge, Wilberforce Road, Cambridge CB3 OWA, United Kingdom \\ Ben Gripaios $\odot^{\dagger}$ and Joseph Tooby-Smith $\odot^{\ddagger}$ \\ Cavendish Laboratory, University of Cambridge, J. J. Thomson Avenue, Cambridge CB3 OHE, United Kingdom
}

(Received 11 February 2020; accepted 19 March 2020; published 8 April 2020)

\begin{abstract}
We consider local (or perturbative) gauge anomalies in models which extend the rank of the Standard Model (SM) gauge group and the chiral fermion content only by $n$ SM singlets. We give a general solution to the anomaly cancellation conditions (ACCs) of an additional $U(1)$ subgroup for the ACCs that involve only SM fermions and we examine whether a corresponding solution exists for the remaining ACCs. We show that a solution to the remaining ACCs always exists for $n \geq 5$ in the familynonuniversal case or $n \geq 3$ in the family-universal case. In the special case where only a single family carries nonvanishing charges, we find a general solution to all ACCs, for any value of $n$.
\end{abstract}

DOI: $10.1103 /$ PhysRevD.101.075015

\section{INTRODUCTION}

Countless gauge extensions of the Standard Model (SM) have been constructed in the literature. Most of these involve an increase in rank with respect to the usual SM gauge group, ${ }^{1}$ so there is usually at least one additional, nonlinearly realized $U(1)$ subgroup in the extension. But if one wishes to tinker with the gauge structure of the SM in this way, one should check that basic requirements of locality and unitarity of the theory are not violated via anomalies in the fermionic path integral.

Our goal here is to carry out the necessary due diligence ${ }^{2}$ on the local $\operatorname{part}^{3}$ of the anomaly. Apart from one crucial

\footnotetext{
*B.C.Allanach@damtp.cam.ac.uk gripaios@hep.phy.cam.ac.uk

\#jss85@cam.ac.uk

${ }^{1}$ An obvious, rare, exception is the $S U(5)$ grand unified theory.

${ }^{2}$ It is well known that local anomalies place nontrivial constraints upon representations of chiral fermions. For example, in the SM, if we allow the hypercharges of the fermions to vary over the reals, the combination of gauge and gravitational anomaly cancellation implies that the charges must be commensurate [1]. Conversely, if the hypercharges are commensurate but otherwise free, gauge anomaly cancellation of a family of SM chiral fermions implies gravitational anomaly cancellation [2] within that family.

${ }^{3}$ The global part is somewhat tricky to study in such models, not least because we do not know (though some might say we do not care) what the gauge group is (see $[3,4]$ for details).
}

Published by the American Physical Society under the terms of the Creative Commons Attribution 4.0 International license. Further distribution of this work must maintain attribution to the author(s) and the published article's title, journal citation, and DOI. Funded by SCOAP. detail (to which we return shortly), the local anomaly depends only on the Lie algebra which, when the rank increases by one, is unambiguously isomorphic to $s u(3) \oplus s u(2) \oplus u(1) \oplus u(1) .^{4}$

The main purpose of our work is to study and restrict the parameter space of additional- $u(1)$ charges of chiral fermions, thus informing the model building of SM extensions.

The gauged, spontaneously broken $U(1)$ subgroup leads to a massive SM-neutral spin-1 particle which we may dub a $Z^{\prime}$. Models with $Z^{\prime}$ have been studied exhaustively in the literature: to explain dark matter [6-13], the anomalous magnetic moment of the muon [14], axions [15] or leptogenesis [16], proton stabilization [17], supersymmetry breaking [18], fermion masses and mixing (via the Froggatt-Nielsen mechanism) [19], and, most recently [8,20-57], apparent lepton family nonuniversality (FNU) in certain rare neutral current $B$-meson decays [58-60]. Several of these applications require FNU couplings of the $Z^{\prime}$, corresponding to FNU charge assignments to its underlying $U(1)$ gauge group. There is also a generic phenomenological motivation for $Z^{\prime}$ fields as follows: the existence of neutrinos and dark matter, among other things, motivates the existence of a rich hidden sector, and it is natural to wonder whether this hidden sector, just like the visible sector, has a gauge symmetry of its own. If that gauge

\footnotetext{
${ }^{4}$ When the rank increases by more than one, this is a subalgebra and all of the considerations in this work remain applicable, but there will, of course, be yet further constraints. For a concrete example in which two additional $U(1)$ factors are gauged, viz. those corresponding to baryon and lepton numbers, see [5].
} 
symmetry algebra has a $u(1)$ factor, then the SM fermions can be charged with respect to it, giving us a possible "portal" to the hidden sector.

Returning now to our discussion of the algebra $s u(3) \oplus s u(2) \oplus u(1) \oplus u(1)$, we can choose a basis $X$, $Y$ for the subalgebra $u(1) \oplus u(1)$ in which $Y$ corresponds to the SM hypercharge and $X$ to the $Z^{\prime}$, such that the $X$ anomaly cancellation conditions (ACCs) become ${ }^{5}$

$$
\begin{gathered}
3^{2} X: 0=\sum_{j=1}^{3}\left(2 Q_{j}+U_{j}+D_{j}\right), \\
2^{2} X: 0=\sum_{j=1}^{3}\left(3 Q_{j}+L_{j}\right), \\
Y^{2} X: 0=\sum_{j=1}^{3}\left(Q_{j}+8 U_{j}+2 D_{j}+3 L_{j}+6 E_{j}\right), \\
Y X^{2}: 0=\sum_{j=1}^{3}(1 \mathrm{a}) \\
\left.\operatorname{grav}^{2} X: \sum_{i=1}^{n} x_{i}=-2 U_{j}^{2}+D_{j}^{2}-L_{j}^{2}+E_{j}^{2}\right),
\end{gathered}
$$

$X^{3}: \sum_{i=1}^{n} x_{i}^{3}=-\sum_{j=1}^{3}\left(6 Q_{j}^{3}+3 U_{j}^{3}+3 D_{j}^{3}+2 L_{j}^{3}+E_{j}^{3}\right)$,

where $F_{j}$ denote the charges of SM fermions ${ }^{6}(F \in\{Q, U$, $D, L, E\}, j \in\{1,2,3\})$ with respect to the $Z^{\prime}$ and $x_{i}$ denote the charges of $n \geq 0$ hypothetical SM-singlet fermions with respect to the $Z^{\prime}$. We posit that $\mathrm{SM}$-singlet fermions are highly likely to exist in nature given the fact that neutrinos are massive and the simplest way of providing neutrino masses is via Yukawa terms with SM-singlet fermions (they also have a number of other phenomenological applications, such as dark matter). By way of shorthand, we refer to the $F_{j}$ as "visible charges" and the $x_{i}$ as "invisible charges." Most previous studies of these ACCs have made additional assumptions, those of family universality or two zero-charged families being particularly common. We wish our analysis to be more general, ultimately allowing the visible charges to freely vary between generations. This will lead to a larger set of solutions that contains subsets with additional assumptions. Aside from being more

\footnotetext{
${ }^{5}$ Given that the symmetry corresponding to the $Z^{\prime}$ is nonlinearly realized, there is always the possibility that the anomalies from chiral fermions do not vanish but are rather compensated by, e.g., a Wess-Zumino-Witten term. We ignore this possibility here.

${ }^{6}$ As usual, we consider all Weyl fermions as being left-handed.
}

general, family dependence is often desirable in applications to flavor physics.

Now comes the crucial point at which the global structure of the gauge group plays a rôle. In the general case, the charges must be real, but if the group is compact (as we might expect on the basis of a variety of theoretical arguments and empirical observations, the most compelling of which are perhaps the apparent unification of gauge couplings and the fact that the observed electric charges themselves appear to be commensurate), then the $X$ charges must be commensurate, and since (1a)-(1f) are invariant under an overall real rescaling, we may take them to be rational.

Of course, by clearing denominators, we could also take them to be coprime integers ${ }^{7}$ and indeed we will always write charges in this way. Thus, for example, we write the SM fermion's hypercharges as $y_{Q_{j}}=+1, y_{U_{j}}=-4$, $y_{D_{j}}=+2, y_{L_{j}}=-3$, and $y_{E_{j}}=+6$. Doing so avoids having to worry about annoying normalization factors. ${ }^{8}$ But considering them to lie in the field of rational numbers allows us to benefit from the not inconsiderable machinery, and geometric insights, of (projective) algebraic geometry. Indeed, the six equations (1) are homogeneous in $15+n$ unknowns with coefficients in $\mathbb{Q}$ and so, given any field extension $k$ of $\mathbb{Q}$ (such as $\mathbb{Q}, \mathbb{R}$, or $\mathbb{C}$ ), they define a projective variety in the $14+n$-dimensional projective space over $k$, i.e., the space of lines through the origin in the affine space $k^{15+n}$.

The holy grail would be to find all $k$ points for each value of $n$. For generic polynomial equations, this is hard enough even for the case $k=\mathbb{R}$ (corresponding to a noncompact gauge group); in the case $k=\mathbb{Q}$ of interest to us (corresponding to a compact gauge group), the best efforts of number theorists over the millennia have yielded scant reward. ${ }^{9}$

The outlook as regards the search for the holy grail is thus somewhat bleak. Fortunately (or unfortunately, depending on one's point of view), a general solution is hardly required, given that so little is known about SMsinglet fermions. It seems likely that they exist (given that

\footnotetext{
${ }^{7}$ That is, integers whose greatest common divisor is 1 .

${ }^{8}$ It also allows us to mention another subtle point. Depending on the choice of the global gauge group, not all rational points lead to admissible representations. Suppose, for example, that the $Z^{\prime}$ together with the $W$ and $Z$ gauge bosons actually make up the group $U(2)$. In this case, the usual global Witten anomaly of $S U(2)$ is absent and appears instead as a local anomaly. The number of even-dimensional representations of $U(2)$ must be even, and if it is nonzero, this implies that the number of odd integer charges must be even; for details, see [3,4]. We will concentrate here on the case in which the global group is assumed to be $S U(3) \times S U(2) \times U(1) \times U(1)$ (which is universal in the sense that it covers any other compact group with the same algebra), such that all rational points are allowed.

${ }^{9}$ The state of the art is elliptic curves, described by a single cubic equation in $2 \mathrm{D}$.
} 
neutrinos are massive), but we do not know how many there are (two or more suffice to fit neutrino data), and we are certainly not yet in a position to measure their charges with respect to a $Z^{\prime}$ boson which is itself yet to be discovered and may well not exist. So, at least for the time being, it seems reasonable to leave the number theorists in peace in their ivory towers and to focus our attention instead on questions which are of more immediate interest to phenomenologists. Happily, we will find that most such questions are also easy enough for phenomenologists to answer.

Paramount among such questions, in our opinion, is the following: can we find all possible values of the visible charges $F_{j}$ for which there exists a solution of the ACCs (1) for some $n$ and $x_{i}$ ? We may wish to know the visible charges because these largely determine the phenomenology of the $Z^{\prime}$. On the other hand, since $n$ and $x_{i}$ are almost completely unconstrained by observations, we hardly need to know the values they take in particular solutions; rather, we might care only whether such values exist.

To this end, our strategy is to first give a general solution of the equations, namely (1a)-(1d), that only involve the visible charges $F_{j}$. The first three of these equations are linear and are thus trivial to solve over $\mathbb{Q}$. The fourth and last equation is quadratic and finding its general solution is also a triviality (albeit an algebraically unpleasant one), once we employ some insight from geometry. Indeed, suppose that we are somehow able to find just one rational point. We may then construct all rational lines through that point. Each such line must either lie in the surface, in which case every rational point on it gives another solution, or it must intersect the surface in another rational point, ${ }^{10}$ giving us a way to generate new solutions from old ones. Moreover, since any two points in (either affine or projective) space are joined by a line, all solutions can be obtained in this way.

The two equations which remain, (1e) and (1f), involve the invisible charges and involve a cubic, so are typically much harder to solve. Instead we attack the simpler problem of fixing $n$ and asking whether a solution exists. To this end, it is convenient to write (1e) and (1f) in the form

$$
\begin{gathered}
\sum_{i=1}^{n} x_{i}=J, \\
\sum_{i=1}^{n} x_{i}^{3}=M+J^{3},
\end{gathered}
$$

where

$$
J:=-\sum_{j=1}^{3}\left(6 Q_{j}+3 U_{j}+3 D_{j}+2 L_{j}+E_{j}\right),
$$

\footnotetext{
${ }^{10}$ This point may, of course, coincide with the original pointi.e., we have a double point-or the other point of intersection may be "at infinity" in affine space.
}

$M:=-\sum_{j=1}^{3}\left(6 Q_{j}^{3}+3 U_{j}^{3}+3 D_{j}^{3}+2 L_{j}^{3}+E_{j}^{3}\right)-J^{3}$,

and evidently $M, J \in \mathbb{Z}$. In the case where two families of charges are set to zero, we will see that $M=0$ and that this allows us to solve (2) and (3) generally. The form of $J$ in this case allows us to connect the solution to this problem to the solution obtained for the visible charges, allowing the general solution to the full set of ACCs to be found.

In more general cases, including the apparently very similar family-universal (FU) case, we are not able to obtain a general solution. But in this FU case, we show in Sec. III that (1e) and (1f) always have a solution over $\mathbb{Z}$ for $n \geq 3$. In the fully family-nonuniversal (FNU) case, we similarly show in Sec. IV D that (1e) and (1f) always have a solution for $n \geq 5$. Thus, that any of the solutions to (1a)-(1d) can be extended to a solution of the full set of ACCs (1a)-(1f) when $n$ is large enough. In contrast, we shall show that sometimes a solution cannot be found for $n \leq 2$ in the FU case and $n \leq 4$ in the FNU case. We catalog the values of $M$ and $J$ for which solutions can be found for $n \leq 2$ in the FU case and $n \leq 3$ in the FNU case. ${ }^{11}$ These results hinge crucially on the not-so-obvious fact that $M \in 6 \mathbb{Z}$. For all cases where a solution exists, we provide a general parameterization of the visible charges.

Thus, we obtain a factorization of the problem in a way that ought to be adequate for phenomenologists' needs: a general parametric solution to the allowed visible charges is given; for any such solution one can be sure that a suitable set of invisible charges exists, if $n$ is large enough.

In an "anomaly-free atlas" [61], solutions of the ACCs considered here were found numerically for $\left|F_{j}\right| \leq 10$ and $n=0,1,2$ or 3 . In the present paper, we are interested in analytic solutions without any such restrictions upon $F_{j}$.

The outline of our paper is as follows. We begin in Sec. II by considering the simple case where only one family is charged. Here we will show that $M=0$, and as a consequence the ACCs can be solved exactly, using previously known results. In Sec. III, we discuss the FU case. In Sec. IV, we discuss the more general case where visible charges vary between generations. We summarize and conclude in Sec. V. Though it is something of a curiosity, it turns out that one can also find a general solution for odd $n$ in the family-universal case; this solution is given in Appendix A. In Appendix B, we supply additional parameterizations of the solutions for the visible charges which avoid the need to consider the degenerate cases where our main parameterization yields entire lines of solutions. The special case where there are only two independent families of charges (which may be

\footnotetext{
${ }^{11}$ There is a small lacuna in the case $n=4$, which can be traced back to the difficulty of solving a cubic equation in one unknown over $\mathbb{Z}$.
} 
phenomenologically relevant given the apparent similarity of the two light SM families) is dealt with in Appendix C.

\section{ONE-FAMILY CASE}

The simplest case we examine is where two families have zero charge and only one family is charged. As such we have $F_{2}=F_{3}=0$ and define $F_{1}=F$. We start our analysis by imposing (1a)-(1c), which imply

$D=-2 Q-U, \quad L=-3 Q, \quad E=2 Q-U$.

Substituting this into (1d) yields $0 .{ }^{12}$ Thus (6) provides a general solution to the visible ACCs (1a)-(1d) for any $Q, U \in \mathbb{Z}$.

Substituting (6) into (4) and (5) gives $J=4 Q+U$ and $M=0$ and so we reduce (2) and (3) to

$$
\sum_{i=1}^{n} x_{i}=J, \quad \sum_{i=1}^{n} x_{i}^{3}=J^{3}
$$

For $n=0$, there exists a solution to all ACCs iff $J=0$, corresponding to a one-parameter family of solutions given by (6) with $U=-4 Q$ already found in Ref. [61]. For $n>0$, by defining $x_{n+1}=-J$, we obtain a set of diophantine equations equivalent to the ACCs of a pure $U(1)$ gauge theory, the general solution to which is known $[62,63]$, and which parameterize $x_{i}$ in terms of a set of parameters $R$ (say). Thus in this case the general solution to the ACCs can be found in terms of the parameters $\{R, Q\}$ :

$U=-4 Q-x_{n+1}(R), \quad D=2 Q+x_{n+1}(R), \quad L=-3 Q$,

$E=6 Q+x_{n+1}(R), \quad x_{i}=x_{i}(R) \quad \forall 1 \leq i \leq n$.

\section{FAMILY-UNIVERSAL CASE}

The next step in our discussion is the FU case. Like for the one-family case above, we start our analysis by imposing (1a)-(1c), leading to (6) again, but where $F_{1}=F_{2}=F_{3}=F$. $^{13}$

\footnotetext{
${ }^{12}$ This has a geometric explanation and holds for generic choices of representations of the five fermionic species. Equation (6) defines a line $L$ in $P \mathbb{Q}^{4}$, which must pass through the point corresponding to hypercharge assignments. The quadratic volume defined by (1d) must also pass through the hypercharge assignment point and so it either must intersect $L$ at one other point or $L$ must lie within the quadratic volume itself. Since (1c) implies that the gradient of (1d) in the direction of the hypercharge point is zero, $L$ lies within the quadratic volume. This argument would not work for a larger number of fermionic species, since then (6) would define a higher-dimensional space rather than a line.

${ }^{13}$ This corresponds to the well-known (see e.g., [1]) fact that, given the $S U(3) \times S U(2)$ representation content of the $\mathrm{SM}$ fermions, the only gaugeable, FU $U(1)$ charges are a linear combination of the usual hypercharge and $B-L$.
}

Substituting (6) into (4), (5) gives us $J=3(4 Q+U)$ and $M=-24(4 Q+U)^{3}$. Notice, here is where our analysis diverges from the one-family case. The fact that $M \neq 0$ prevents us from solving (2) and (3) in a way identical to $[62,63]$. As such we are left to study (2) and (3) on a case-bycase basis, looking at the existence of solutions. For $n=0$, it is obvious that we need $J=0 \Rightarrow M=0$, leading to the same one-parameter set of solutions as in the $n=0$ one-family case. For $n=1, x_{1}=J$ and $x_{1}^{3}=M+J^{3}$, so $M=0 \Rightarrow J=0$ as well. For $n=2, x_{1}+x_{2}=J$ and $x_{1}^{3}+x_{2}^{3}=M+J^{3}$, eliminating $x_{2}$ from the latter equation gives

$$
9 K x_{1}^{2}-27 K^{2} x_{1}+24 K^{3}=0,
$$

where $K \equiv J / 3 \in \mathbb{Z}$ and we have used that $M=-24 K^{3}$. This equation has no real roots (ergo no integer roots) unless $K=0 \Rightarrow J=M=0$ again. For $n \geq 3$, we always have a solution given by $x_{1}=x_{2}=x_{3}=K=(4 Q+U)$ and $x_{i}=0$ for $i>3$. While this is $a$ solution, we do not claim that it is the most general one. However, in the case of $n$ odd it is an oddity that we can find the most general solution. ${ }^{14}$ This is detailed in Appendix A.

We will see in Sec. IV D that we will be less lucky when considering the equivalent problem in the FNU case, and we will have to resort to less trivial techniques.

\section{FAMILY-NONUNIVERSAL CASE}

We now move to the FNU case and find ourselves in the fortunate situation that the ACCs (1a)-(1d) (those that depend only on visible matter) can, when considered alone, be solved generally using straightforward, if unpleasant, techniques from diophantine analysis. To do so, it helps to apply a common $G L(3, \mathbb{Z})$ transformation to $\left\{F_{1}, F_{2}, F_{3}\right\}$ similar to that in Ref. [61], so as to recast the equations into a simpler form, while remaining within the realm of the integers.

To wit, we set $F_{+}=F_{1}+F_{2}+F_{3}, F_{\alpha}=F_{1}-F_{2}$, and $F_{\beta}=F_{2}+F_{3}$. The judiciousness of this transformation is twofold. Firstly, the linear equations (1a)-(1c) become dependent on $F_{+}$only, whose general solution over $\mathbb{Z}$ is immediately seen to be

$$
\begin{aligned}
D_{+} & =-2 Q_{+}-U_{+}, \quad L_{+}=-3 Q_{+}, \\
E_{+} & =2 Q_{+}-U_{+},
\end{aligned}
$$

written in term of two arbitrary parameters $Q_{+}, U_{+} \in \mathbb{Z}$, which we will see are further constrained by the quadratic. Secondly, it makes it easy for us to recover the results in situations with charge universality between any two families, which without loss of generality corresponds to setting $F_{\alpha}=0$; this is considered in Appendix C.

\footnotetext{
${ }^{14}$ It arises because the cubic hypersurface has double points, and so all solutions can be obtained by constructing lines through such a point.
} 
In the new variables the quadratic equation becomes

$$
X^{T} H X=0,
$$

where we have used (10) to replace $D_{+}, L_{+}$and $E_{+}$. It is a homogenous diophantine equation of degree 2 in the entries of the 12-tuple

$$
X:=\left(Q_{+}, U_{+}, Q_{\alpha}, Q_{\beta}, U_{\alpha}, U_{\beta}, D_{\alpha}, D_{\beta}, L_{\alpha}, L_{\beta}, E_{\alpha}, E_{\beta}\right)
$$

$H$ is a $12 \times 12$ symmetric matrix with integer entries, the upper right triangle of which is

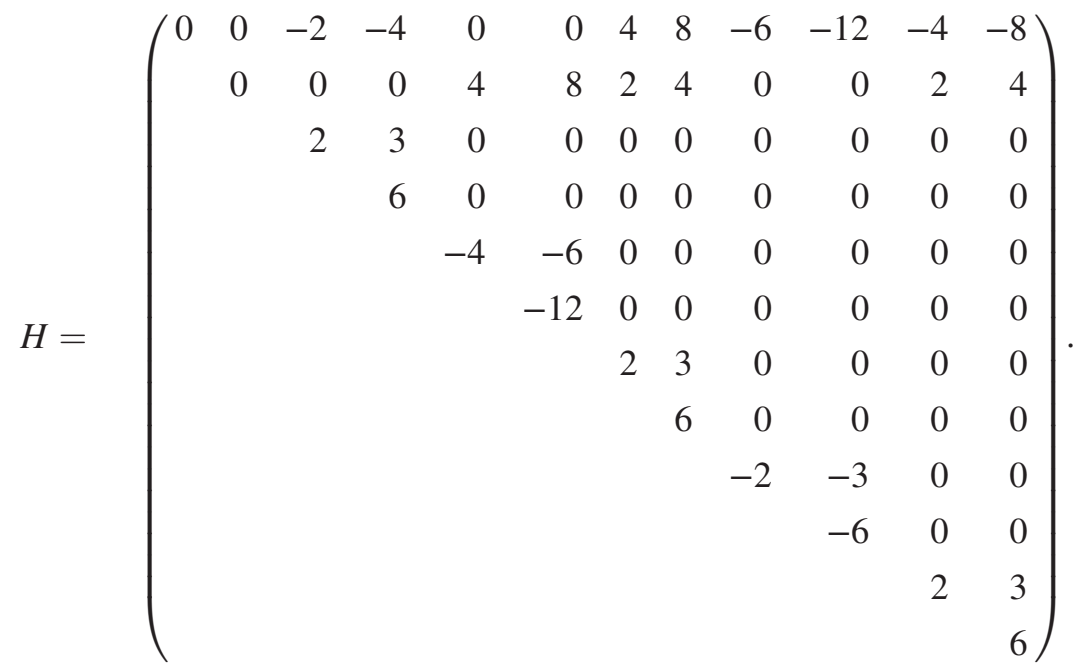

We shall now show that (11), which defines a hypersurface $\Gamma \subset \mathbb{P Q}^{11}$, can be solved generally over $\mathbb{Z}$ given only one nontrivial solution. We consider lines $L=\alpha \tilde{X}+\beta R$ through a known solution $\tilde{X} \in \mathrm{PQ}^{11}$, where $R \in \mathrm{PQ}^{11}$, and $[\alpha: \beta] \in \mathbb{P Q}^{1}$. On substitution into (11) we obtain

$$
\beta\left(2 R^{T} H \tilde{X} \alpha+R^{T} H R \beta\right)=0
$$

Thus either the line $L$ intersects $\Gamma$ at $\beta=0$ (returning the point $\tilde{X}$ ) and at $[\alpha: \beta]=\left[R^{T} H R:-2 R^{T} H \tilde{X}\right]$, or $L \subset \Gamma$ with (14) being automatically satisfied for any $\alpha$ and $\beta$.

For $L \nsubseteq \Gamma$, after returning to affine space, the solutions generated from the second intersection point are

$$
X=\frac{k}{\operatorname{GCD}\left(X^{\prime}\right)} X^{\prime},
$$

where $X^{\prime}=\left(R^{T} H R\right) \tilde{X}-2\left(R^{T} H \tilde{X}\right) R$,

where $k \in \mathbb{Z}$ is an overall factor and $\operatorname{GCD}\left(X^{\prime}\right)$ denotes the greatest common divisor of the integers in $X^{\prime}$.

\section{A. Solution for SM chiral fermion charges}

A specific choice of $\tilde{X}$ has $Q_{\alpha}=1$ and $L_{\alpha}=1$, with all other parameters zero. Using the formula (15) we obtain

$$
\begin{array}{rlrl}
Q_{+}^{\prime} & =2 R_{Q_{+}} \Lambda, & U_{+}^{\prime}=2 R_{U_{+}} \Lambda, & Q_{\alpha}^{\prime}=2 R_{Q_{\alpha}} \Lambda+\Sigma, \\
Q_{\beta}^{\prime}=2 R_{Q_{\beta}} \Lambda, & U_{\alpha}^{\prime}=2 R_{U_{\alpha}} \Lambda, & U_{\beta}^{\prime}=2 R_{U_{\beta}} \Lambda, \\
D_{\alpha}^{\prime}=2 R_{D_{\alpha}} \Lambda, & D_{\beta}^{\prime}=2 R_{D_{\beta}} \Lambda, & L_{\alpha}^{\prime}=2 R_{L_{\alpha}} \Lambda+\Sigma, \\
L_{\beta}^{\prime}=2 R_{L_{\beta}} \Lambda, & E_{\alpha}^{\prime}=2 R_{E_{\alpha}} \Lambda, & E_{\beta}^{\prime}=2 R_{E_{\beta}} \Lambda, \quad(16)
\end{array}
$$

where

$$
\begin{gathered}
\Lambda=\left(8 R_{Q_{+}}+2 R_{L_{\alpha}}+3 R_{L_{\beta}}-2 R_{Q_{\alpha}}-3 R_{Q_{\beta}}\right), \\
\Sigma=R^{T} H R .
\end{gathered}
$$

We invert the $G L(3, \mathbb{Z})$ transformation used above using (10) and (16) to yield a parameterization of a solution for the visible charges 


$$
\begin{aligned}
& Q_{1}=2 \Lambda\left(R_{Q_{+}}-R_{Q_{\beta}}\right), \\
& Q_{2}=2 \Lambda\left(R_{Q_{+}}-R_{Q_{\alpha}}-R_{Q_{\beta}}\right)-\Sigma, \\
& Q_{3}=2 \Lambda\left(2 R_{Q_{\beta}}+R_{Q_{\alpha}}-R_{Q_{+}}\right)+\Sigma, \\
& U_{1}=2 \Lambda\left(R_{U_{+}}-R_{U_{\beta}}\right), \\
& U_{2}=2 \Lambda\left(R_{U_{+}}-R_{U_{\alpha}}-R_{U_{\beta}}\right), \\
& U_{3}=2 \Lambda\left(2 R_{U_{\beta}}+R_{U_{\alpha}}-R_{U_{+}}\right), \\
& D_{1}=-2 \Lambda\left(2 R_{Q_{+}}+R_{U_{+}}+R_{D_{\beta}}\right), \\
& D_{2}=-2 \Lambda\left(R_{D_{\alpha}}+R_{D_{\beta}}+2 R_{Q_{+}}+R_{U_{+}}\right), \\
& D_{3}=2 \Lambda\left(R_{D_{\alpha}}+2 R_{D_{\beta}}+2 R_{Q_{+}}+R_{U_{+}}\right), \\
& L_{1}=-2 \Lambda\left(R_{L_{\beta}}+3 R_{Q_{+}}\right), \\
& L_{2}=-2 \Lambda\left(R_{L_{\alpha}}+R_{L_{\beta}}+3 R_{Q_{+}}\right)-\Sigma, \\
& L_{3}=2 \Lambda\left(R_{L_{\alpha}}+2 R_{L_{\beta}}+3 R_{Q_{+}}\right)+\Sigma, \\
& E_{1}=2 \Lambda\left(2 R_{Q_{+}}-R_{E_{\beta}}-R_{U_{+}}\right), \\
& E_{2}=2 \Lambda\left(2 R_{Q_{+}}-R_{E_{\alpha}}-R_{E_{\beta}}-R_{U_{+}}\right), \\
& E_{3}=2 \Lambda\left(-2 R_{Q_{+}}+R_{E_{\alpha}}+2 R_{E_{\beta}}+R_{U_{+}}\right)
\end{aligned}
$$

in terms of the 12 integer-valued variables

$$
\begin{aligned}
& \left\{R_{Q_{+}}, R_{U_{+}}, R_{Q_{\alpha}}, R_{Q_{\beta}}, R_{U_{\alpha}}, R_{U_{\beta}}, R_{D_{\alpha}}, R_{D_{\beta}},\right. \\
& \left.\quad R_{L_{\alpha}}, R_{L_{\beta}}, R_{E_{\alpha}}, R_{E_{\beta}}\right\} .
\end{aligned}
$$

Note that, for this $\tilde{X}$, those solutions for which $Q_{\alpha}=L_{\alpha}$ lie on lines which themselves lie in $\Gamma$. These points can be caught by either scanning over all $R$ and noting that when $R^{T} H \tilde{X}=0$ all rational points on the line are solutions or by taking a series of different $\tilde{X}$. In Appendix B we give a set of $\tilde{X}$, such that all solutions can be found as the unique second intersection point of $\Gamma$ with a line through $\tilde{X}$.

\section{B. Gauge-invariant Yukawa couplings}

As an example of how the parameterization in (19) could be used, we ask the question: what are conditions on $R$ from the existence of Yukawa couplings?

First, we note that a necessary and sufficient condition for the presence of all possible gauge-invariant Yukawa couplings is that we have family universality, as shown in Ref. [61]. We analyzed this case in Sec. III. Going to the family-nonuniversal case, we write the Yukawa couplings in the Lagrangian density:

$\mathcal{L}_{Y}=\left(Y_{U}\right)_{i j} q_{i} H^{c} u_{j}^{c}+\left(Y_{D}\right)_{i j} q_{i} H d_{j}^{c}+\left(Y_{E}\right)_{i j} l_{i} H e_{j}^{c}+$ H.c.,

where $\left\{q_{i}, u_{i}^{c}, d_{i}^{c}, l_{i}, e_{i}^{c}\right\}$ are the left-handed fermion fields in the family indexed by $i, H$ is the SM Higgs doublet field and $\left(Y_{U, D, E}\right)_{i j}$ are 3 by 3 matrices of dimensionless Yukawa couplings for the up quarks, down quarks and charged leptons, respectively. In the SM, the top Yukawa coupling is $\sim \mathcal{O}(1)$, so we require that it is allowed by the additional $U(1)$ symmetry at the unbroken level, i.e.,

$$
H_{0}=Q_{1}+U_{1},
$$

where $H$ is a charge of $H_{0}$ with respect to the $Z^{\prime}$. Identifying the left- and (anti-)right-handed top fields with $Q_{1}$ and $u_{1}^{c}$, we then obtain from (19) the charges for field operators multiplying the Yukawa coupling in Table I. If the charge combination is nonzero, then the Yukawa coupling is absent from the unbroken theory (by gauge symmetry).

We note that there are many different choices that one could make, by identifying the top quark with $Q_{2,3}$ and/or $u_{2,3}$ fields or indeed by choosing one of the other sets of solutions shown in Table III. However, rather than provide an encyclopedia of Yukawa couplings for all possible solutions, our purpose here is to illustrate how our solutions may be used and what some of the issues are.

Since the top, bottom and tau have large masses compared to the other fermions, we might expect each of their Yukawa couplings to be present, being gauge invariant under the additional $U(1)$ subgroup. This subgroup may well ban other tree-level, renormalizable Yukawa couplings, but because the additional $U(1)$ symmetry is spontaneously broken, we expect small corrections to any zeros in the Yukawa matrices. This is the philosophy behind many models of fermion masses, including the third family hypercharge model [49], where such hierarchies are successfully employed to qualitatively explain two features: small quark mixing and the relative heaviness of the third family of fermions. A discussion of neutrino masses requires an analysis involving the SM singlets, whose charges we do not yet have a general solution for, and so we leave these for future work.

Let us, for the sake of illustration, further identify the tau lepton with $L_{1}$ and $e_{1}$ and the bottom quark with $Q_{1}$ and $d_{1}$. By consulting Table I, we see that for the (11) entry of each Yukawa matrix to be allowed under the additional $U(1)$ subgroup,

$$
H_{0}=-Q_{1}-D_{1}=-L_{1}-E_{1},
$$

in addition to (21). Using (19) the additional constraints become requirements on $R$, given by either

$$
\begin{aligned}
& R_{U_{+}}=-R_{D_{\beta}}+R_{E_{\beta}}+R_{L_{\beta}}-R_{Q_{\beta}}-R_{Q_{+}}, \\
& R_{U_{\beta}}=-R_{D_{\beta}}-2 R_{Q_{\beta}},
\end{aligned}
$$

or the trivial solution $\Lambda=0$, which we discount as being uninteresting, having only zero $Z^{\prime}$ charges for the SM fermions. For the nontrivial solution, (23) reduces the dimensionality of the solutions from 12 to 10 . 
TABLE I. $\quad Z^{\prime}$ charges of field operators multiplying each entry of a Yukawa matrix from (19). In the unbroken $U(1)$ theory, the Yukawa term may only be present if the charge is equal to zero. The first column labels the entry of the matrix; the second gives the charge. $\Lambda$ and $\Sigma$ are given in (17) and (18), respectively.

\begin{tabular}{|c|c|}
\hline$i j$ & Charge \\
\hline & $\left(Y_{U}\right)$ \\
\hline 11 & 0 \\
\hline 12 & $-2 \Lambda R_{U_{\alpha}}$ \\
\hline 13 & $2 \Lambda\left(R_{U_{\alpha}}+3 R_{U_{\beta}}-2 R_{U_{+}}\right)$ \\
\hline 21 & $-2 \Lambda R_{Q_{\alpha}}-\Sigma$ \\
\hline 22 & $-2 \Lambda\left(R_{Q_{\alpha}}+R_{U_{\alpha}}\right)-\Sigma$ \\
\hline 23 & $2 \Lambda\left(-R_{Q_{\alpha}}+R_{U_{\alpha}}+3 R_{U_{\beta}}-2 R_{U_{+}}\right)-\Sigma$ \\
\hline 31 & $2 \Lambda\left(R_{Q_{\alpha}}+3 R_{Q_{\beta}}-2 R_{Q_{+}}\right)+\Sigma$ \\
\hline 32 & $2 \Lambda\left(R_{Q_{\alpha}}+3 R_{Q_{\beta}}-2 R_{Q_{+}}-R_{U_{\alpha}}\right)+\Sigma$ \\
\hline 33 & $2 \Lambda\left(R_{Q_{\alpha}}+3 R_{Q_{\beta}}-2 R_{Q_{+}}+R_{U_{\alpha}}+3 R_{U_{\beta}}-2 R_{U_{+}}\right)+\Sigma$ \\
\hline & $\left(Y_{D}\right)$ \\
\hline 11 & $-2 \Lambda\left(R_{D_{\beta}}+2 R_{Q_{\beta}}+R_{U_{\beta}}\right)$ \\
\hline 12 & $-2 \Lambda\left(2 R_{D_{\alpha}}+R_{D_{\beta}}+2 R_{Q_{\beta}}-R_{Q_{+}}+R_{U_{\beta}}\right)$ \\
\hline 13 & $2 \Lambda\left(R_{D_{\alpha}}+2\left(R_{D_{\beta}}-R_{Q_{\beta}}+2 R_{Q_{+}}+R_{U_{+}}\right)-R_{U_{\beta}}\right)$ \\
\hline 21 & $-2 \Lambda\left(R_{D_{\beta}}+R_{Q_{\alpha}}+2 R_{Q_{\beta}}+R_{U_{\beta}}\right)-\Sigma$ \\
\hline 22 & $-2 \Lambda\left(2 R_{D_{\alpha}}+R_{D_{\beta}}+R_{Q_{\alpha}}+2 R_{Q_{\beta}}-R_{Q_{+}}+R_{U_{\beta}}\right)-\Sigma$ \\
\hline 23 & $2 \Lambda\left(R_{D_{\alpha}}+2 R_{D_{\beta}}-R_{Q_{\alpha}}-2 R_{Q_{\beta}}+4 R_{Q_{+}}-R_{U_{\beta}}+2 R_{U_{+}}\right)-\Sigma$ \\
\hline 31 & $-2 \Lambda\left(R_{D_{\beta}}-R_{Q_{\alpha}}-R_{Q_{\beta}}+2 R_{Q_{+}}+R_{U_{\beta}}\right)+\Sigma$ \\
\hline 32 & $-2 \Lambda\left(2 R_{D_{\alpha}}+R_{D_{\beta}}-R_{Q_{\alpha}}-R_{Q_{\beta}}+R_{Q_{+}}+R_{U_{\beta}}\right)+\Sigma$ \\
\hline 33 & $2 \Lambda\left(R_{D_{\alpha}}+2 R_{D_{\beta}}+R_{Q_{\alpha}}+R_{Q_{\beta}}+2 R_{Q_{+}}-R_{U_{\beta}}+2 R_{U_{+}}\right)+\Sigma$ \\
\hline & $\left(Y_{E}\right)$ \\
\hline 11 & $-2 \Lambda\left(R_{E_{\beta}}+R_{L_{\beta}}+R_{Q_{\beta}}+R_{U_{\beta}}\right)$ \\
\hline 12 & $-2 \Lambda\left(R_{E_{\alpha}}+R_{E_{\beta}}+R_{L_{\beta}}+R_{Q_{\beta}}+R_{U_{\beta}}\right)$ \\
\hline 13 & $-2 \Lambda\left(-R_{E_{\alpha}}-2 R_{E_{\beta}}+R_{L_{\beta}}+R_{Q_{\beta}}+4 R_{Q_{+}}+R_{U_{\beta}}-2 R_{U_{+}}\right)$ \\
\hline 21 & $-2 \Lambda\left(R_{E_{\beta}}+R_{L_{\alpha}}+2 R_{L_{\beta}}+R_{Q_{\beta}}+R_{U_{\beta}}\right)-\Sigma$ \\
\hline 22 & $-2 \Lambda\left(R_{E_{\alpha}}+R_{E_{\beta}}+R_{L_{\alpha}}+2 R_{L_{\beta}}+R_{Q_{\beta}}+R_{U_{\beta}}\right)-\Sigma$ \\
\hline 23 & $-2 \Lambda\left(-R_{E_{\alpha}}-2 R_{E_{\beta}}+R_{L_{\alpha}}+2 R_{L_{\beta}}+R_{Q_{\beta}}+4 R_{Q_{+}}+R_{U_{\beta}}-2 R_{U_{+}}\right)-\Sigma$ \\
\hline 31 & $-2 \Lambda\left(R_{E_{\beta}}+R_{L_{\alpha}}+R_{L_{\beta}}+R_{Q_{\beta}}+R_{U_{\beta}}\right)+\Sigma$ \\
\hline 32 & $-2 \Lambda\left(R_{E_{\alpha}}+R_{E_{\beta}}+R_{L_{\alpha}}+R_{L_{\beta}}+R_{Q_{\beta}}+R_{U_{\beta}}\right)+\Sigma$ \\
\hline 33 & $2 \Lambda R_{E_{\alpha}}+4 \Lambda R_{E_{\beta}}-2 \Lambda\left(R_{L_{\alpha}}+R_{L_{\beta}}+R_{Q_{\beta}}+4 R_{Q_{+}}+R_{U_{\beta}}-2 R_{U_{+}}\right)+\Sigma$ \\
\hline
\end{tabular}

\section{A lemma on $M$}

Here we show that $M \in 6 \mathbb{Z}$ [see (5) for the definition of $M]$, a result that will be of use later. From (1a) we have $\sum_{j}^{3}\left(U_{j}+D_{j}\right)=0 \bmod 2$ and from (1b) we have $\sum_{j}^{3} L_{j}=0 \bmod 3$ which also imply that $\sum_{j}^{3}\left(U_{j}^{3}+D_{j}^{3}\right)=$ $0 \bmod 2$ and $\sum_{j}^{3} L_{j}^{3}=0 \bmod 3$, respectively. Hence $J=-\sum_{j}^{3} E_{j} \bmod 6$ and $M+J^{3}=-\sum_{j}^{3} E_{j}^{3} \bmod 6$, which gives us $M=3\left(E_{1}+E_{2}\right)\left(E_{2}+E_{3}\right)\left(E_{3}+E_{1}\right) \bmod 6$. Since $\left(E_{1}+E_{2}\right)\left(E_{2}+E_{3}\right)\left(E_{3}+E_{1}\right)$ is always even for any integers $E_{i}$, we have $M=0 \bmod 6$ QEDGiven this it is convenient for us to define $6 P=M$.

\section{The number of SM singlets}

Although we were lucky with the visible ACCs, allowing us to solve them generally, we will be less lucky with the remaining two ACCs (2) and (3) which describe the invisible charges. As such, we will proceed in a similar manner to the FU case and establish the following facts: for $n \leq 4$ a solution does not always exist; for $n \geq 5$ a solution always exists. Furthermore, for $n \leq 3$ we give a complete characterization of the solutions. Let us begin with a discussion of these cases.

\section{1. $n \leq 3$}

For $n=0$, the ACCs clearly have a solution iff $P=J=0$. For any $n \geq 1$, we can eliminate $x_{n}$ from (2) and (3); the resulting equation, which must be symmetric under permutations of the remaining $n-1$ charges, may be recast in terms of the elementary symmetric polynomials $e_{j}\left(x_{1}, \ldots, x_{n-1}\right) \equiv \sum_{1 \leq i_{1}<i_{2}<\cdots<i_{j} \leq n-1} x_{i_{1}} x_{i_{2}} \ldots x_{i_{j}}$ as 


$$
e_{3}=2 P+e_{1} e_{2}+J e_{1}\left(J-e_{1}\right) .
$$

This condition makes it relatively easy to deduce when we have a solution for $n \leq 3$, as follows.

For $n=1$, we have $e_{1}=e_{2}=e_{3}=0 \Rightarrow P=0$, in which case the solution is $x_{1}=J$.

For $n=2$, we have $e_{2}=e_{3}=0 \Rightarrow 0=2 P+$ $J e_{1}\left(J-e_{1}\right)$. This quadratic equation in $e_{1}$ has a solution in the integers only if $J$ divides $2 P$ (barring the trivial case $J=0$, for which the solution is $x_{1}=-x_{2}$ ). If so, we have the equation $e_{1}^{2}-J e_{1}-2 P / J=0$ with integer coefficients. Since the leading coefficient is unity, any rational solution is also an integer solution. To get a rational solution, the discriminant $J^{2}+8 P / J$ must be square. So we have an integer solution iff $J$ divides $2 P$ and $J^{2}+8 P / J$ is square. (As a check, our result for $n=2$ subsumes the result for $n=1$, since if $P=0$, then $J^{2}+8 P / J=J^{2}$ is certainly square.) The two invisible charges $x_{1}$ and $x_{2}$ are then the two roots of $x^{2}-J x-2 P / J=0$ and if a solution exists, it is unique up to permutation of the charges.

For $n=3$, we have $e_{3}=0 \Rightarrow 2 P+e_{1} e_{2}+$ $J e_{1}\left(J-e_{1}\right)=0$, such that the two charges $x_{1}$ and $x_{2}$ are fixed to be the two roots of the quadratic equation

$$
0=x^{2}-e_{1} x+e_{1} J-2 P / e_{1}-J^{2},
$$

where the third charge is given by $J-e_{1}$. Since both roots $x_{1}$ and $x_{2}$ correspond to charges, they must both be valued in the integers and so must their product, which equals $e_{1} J-2 P / e_{1}-J^{2}$. Thus $e_{1}$ must divide $2 P$. As for $n=2$, any rational solution must then be an integer solution because the leading coefficient is unity, and a rational solution is obtained iff the discriminant $\left(2 J-e_{1}\right)^{2}+$ $8 P / e_{1}$ is square. In toto, we have that there exists a solution iff there exists a divisor $e_{1}$ of $2 P$ such that $\left(2 J-e_{1}\right)^{2}+8 P / e_{1}$ is square. The number of possible solutions is finite (at least for $P \neq 0$ ), being at most (up to permutation of roots) given by the number of divisors of $2 P$.

To recover the result for $n=2$, set $e_{1}=J$, such that the third root is 0 . Then we have a solution iff $J^{2}+8 P / J$ is square, such that $J$ divides $2 P$, which are precisely the conditions found for $n=2$.

\section{2. $n=4$}

When $n=4$, we can use similar considerations to those for $n \leq 3$ to conclude that the three charges $x_{1}, x_{2}$, and $x_{3}$ should be the three solutions of the cubic

$$
\begin{aligned}
x^{3} & -e_{1} x^{2}+e_{2} x-e_{3} \\
& =x^{3}-e_{1} x^{2}+e_{2} x-\left(2 P+e_{1} e_{2}+J e_{1}\left(J-e_{1}\right)\right)=0 .
\end{aligned}
$$

A necessary (but not sufficient) condition for the charges to be integers is that the discriminant

$$
\begin{gathered}
-\left(e_{1} J\left(J-e_{1}\right)+2 P\right)\left(27 e_{1} J^{2}-27 e_{1}^{2} J+4 e_{1}^{3}+54 P\right) \\
-4 e_{2}^{3}-8 e_{2}^{2} e_{1}^{2}-4 e_{2} e_{1}\left(9 e_{1} J^{2}-9 e_{1}^{2} J+e_{1}^{3}+18 P\right)
\end{gathered}
$$

must be expressible in the form $r^{2} s^{2}(r-s)^{2}$, for some $r, s \in \mathbb{Z}$. Indeed the discriminant is defined to be the product of the squares of the differences of the roots and we are free to shift the roots by an integer such that one root vanishes, without changing the discriminant's value. But this condition is difficult to express in terms of conditions on $P$ and $J$. Thus we content ourselves with showing that there exist $P$ and $J$ for which no solution can be found.

By explicit evaluation of the various possibilities for the various charges modulo 9 , we find the following conditions on $P$ and $J(\bmod 9)$ for a solution to exist:

$J \bmod 9 \in\{1,4,7\}$ requires $P \bmod 9 \notin\{2,5\}$,

$J \bmod 9 \in\{2,5,8\}$ requires $P \bmod 9 \notin\{4,7\}$.

Any of the 12 cases (mod 9) not covered above could furnish us with a counterexample, if we could find corresponding values for the visible charges. A particularly simple counterexample is given by

$$
\begin{array}{lll}
Q_{1}=-2, & Q_{2}=0, & Q_{3}=2, \\
E_{1}=-1, & E_{2}=1, & E_{3}=1, \\
U_{1}=-2, & U_{2}=0, & U_{3}=1, \\
L_{1}=-1, & L_{2}=-1, & L_{3}=2, \\
D_{1}=-1, & D_{2}=0, & D_{3}=2,
\end{array}
$$

which satisfy the ACCs (1a)-(1d) but give $(P, J)=(2,1)$. Thus we see that it is not always possible to find suitable charges for four invisible particles such that all anomalies cancel.

\section{3. $n \geq 5$}

For the case of $n \geq 5$ and for any given $P$ and $J$ there is always a set of invisible charges which satisfy (2) and (3). To show this, we show it is true for the $n=5$ case, with the $n>5$ cases immediately following by setting the extra charges to zero.

For $n=5$, we set $x_{5}=J$ and $x_{4}=-x_{1}-x_{2}-x_{3}$, which immediately satisfies (2) and reduces (3) to

$$
\left(x_{2}+x_{3}\right)\left(x_{3}+x_{1}\right)\left(x_{1}+x_{2}\right)=-2 P .
$$

The choice of charges $x_{1}=P+1, x_{2}=-P$ and $x_{3}=-P$ satisfies the above equation and is integer. Thus the integer set of charges

$$
\{P+1, P-1,-P,-P, J\}
$$

satisfies (2) and (3) for $n=5$.

The results of this section are summarized in Table II. 
TABLE II. Summary of the solutions to (2) and (3) for various $n$ in the FNU case.

\begin{tabular}{|c|c|c|}
\hline$n$ & Solutions exist & Invisible charges are roots $x$ of \\
\hline 0 & iff $J=0$ and $P=0$ & \\
\hline 1 & iff $P=0$ & $x=J$ \\
\hline 2 & iff $J \mid 2 P$ and $J^{2}+8 P / J$ is square & $x^{2}-J x-2 P / J=0$ \\
\hline 3 & iff $\exists e_{1}$ such that $e_{1} \mid 2 P$ and $\left(2 J-e_{1}\right)^{2}+8 P / e_{1}$ is square & $\left(x-J+e_{1}\right)\left(x^{2}-e_{1} x+e_{1} J-2 P / e_{1}-J^{2}\right)=0$ \\
\hline 4 & only if $(P, J) \notin\{(\{2,5\},\{1,4,7\}),(\{4,7\},\{2,5,8\})\}$ & $\left(x-J+e_{1}\right)\left(x^{3}-e_{1} x^{2}+e_{2} x-2 P-e_{1} e_{2}-J e_{1}\left(J-e_{1}\right)\right)=0$ \\
\hline$n \geq 5$ & $\forall P, J$ & e.g., $x^{n-5}(x-J)(x-1-P)(x-P+1)(x+P)^{2}=0$ \\
\hline
\end{tabular}

\section{SUMMARY AND CONCLUSION}

Countless gauge extensions of the SM have been constructed, most of them ${ }^{15}$ including a $U(1)$ subgroup in the extension. In this paper, we have studied possible values of the charges of chiral fermions under the additional $U(1)$ with two assumptions: (i) that local anomalies cancel and (ii) that the chiral fermion content is that of the SM plus a number $n$ of SM-singlet chiral fermions. The ACCs split into two classes: the first class, with four equations, involves no SM-singlet fermionic charges. The second class, with two equations, does involve them. Assumption (ii) is crucial; the assumption of additional SM singlets allows a general solution to the problem, such as it is specified, to be found: the charges of SM chiral fermions under the additional $U(1)$ subgroup are parameterized in (19). Solving for the SM-singlet charges in general from the second class proves to be much trickier. We thus resort to calculating how many of them are required to provide a solution to all of the ACCs.

Before tackling the most general problem, we examine two simpler and well-motivated cases where only one family is charged and where all visible charges are family universal. In the former case we were able to solve the problem in full generality, specifying a parameterization of the solution for the visible and SM-singlet charges (of a specified number). For this case, a solution to the first class of ACCs can always be extended to a full solution of all six ACCs when there are three or more SM singlets.

We then moved onto the full family-nonuniversal case, solving the first class of ACCs in full generality; cf. (19). The result is a 12-dimensional parameterization of SM fermion charges, shown in (19). We have illustrated how the parameterization may be used in practice by examining constraints leading to the presence or absence of Yukawa couplings under the full gauge invariance in Sec. IV B. Requiring gauge-invariant third-family Yukawa couplings restricts the dimensionality of the parameterization further and could be used as a basis for more detailed fermion mass model building.

Our progress for the invisible charges followed a similar path to the family-universal case. We found conditions on

\footnotetext{
${ }^{15}$ One notable exception is $S U(5)$, which has been studied $a d$ nauseam in the literature.
}

functions of $U(1)$ charges of SM fermions $J$ and $M$, such that a solution to the first class of ACCs could be extended to the full ACCs, for up to $n=3 \mathrm{SM}$-singlet charges. We also showed that the minimum number of SM-singlet charges such that a solution can always be extended is 5 . We give a solution for these five singlet charges.

If the SM is extended by some non-Abelian group $G$ with a $U(1)$ gauge subgroup, our analysis obviously still applies to the charges of chiral fermions under the $U(1)$ subgroup. One may take our solution parameterizations and then apply any further constraints implied by the rest of $G$.

\section{ACKNOWLEDGMENTS}

We thank other members of the Cambridge Pheno Working Group for discussions. This work has been partially supported by Science and Technology Facilities Council consolidated Grants No. ST/P000681/1 and No. ST/S505316/1. B. G. is also supported by King's College, Cambridge.

\section{APPENDIX A: SOLUTION FOR INVISIBLE CHARGES FOR THE FAMILY-UNIVERSAL CASE AND $n$ ODD}

In Sec. III we had the equations

$$
\begin{gathered}
\sum_{i=1}^{n} x_{i}=J=3 K, \\
\sum_{i=1}^{n} x_{i}^{3}=J^{3}+M=3 K^{3},
\end{gathered}
$$

where $K=4 Q+U$. It turns out that (A1) and (A2) can be solved exactly for odd $n$. They imply that

$$
9 \sum_{i=1}^{n} x_{i}^{3}-\left(\sum_{i=1}^{n} x_{i}\right)^{3}=0,
$$

defining a cubic surface $C$ in the projective space $\mathrm{PQ}^{n-1}$. This cubic surface has a double point (a point on the surface where all derivatives of the surface vanish) at (for $n \geq 3$ and odd)

$$
\begin{aligned}
S:= & {[1: 1: 1:-1: 1:-1: 1:} \\
& \cdots:-1: 1] \in \mathrm{PQ}^{n-1} .
\end{aligned}
$$


TABLE III. A set of known solutions $\tilde{X}$, which together can generate all nontrivial solutions to $X^{T} H X=0$, along with a condition on $X$ such that $X^{T} H \tilde{X}=0$. If $X$ does not satisfy this condition, then it can be generated by the corresponding $\tilde{X}$.

\begin{tabular}{lcc}
\hline \hline$i$ & Nonzero elements of $\tilde{X}$ & Condition on $X$ for $X^{T} H \tilde{X}=0$ \\
\hline 1 & $Q_{\alpha}=L_{\alpha}=1$ & $Q_{\alpha}=L_{\alpha}$ \\
2 & $D_{\alpha}=L_{\alpha}=1$ & $D_{\alpha}=L_{\alpha}$ \\
3 & $L_{\alpha}=E_{\alpha}=1$ & $E_{\alpha}=L_{\alpha}$ \\
4 & $Q_{\alpha}=U_{\alpha}=D_{\alpha}=L_{\alpha}=E_{\alpha}=1$ & $Q_{\beta}=L_{\beta}$ \\
5 & $Q_{\beta}=L_{\beta}=1$ & $D_{\beta}=L_{\beta}$ \\
6 & $D_{\beta}=L_{\beta}=1$ & $E_{\beta}=L_{\beta}$ \\
7 & $L_{\beta}=E_{\beta}=1$ & $Q_{\alpha}-2 U_{\alpha}+D_{\alpha}-L_{\alpha}$ \\
8 & $Q_{\beta}=D_{\beta}=L_{\beta}=E_{\beta}=1$ & $Q_{\beta}-2 U_{\beta}+D_{\beta}-L_{\beta}+E_{\beta}=0$ \\
9 & $Q_{\alpha}=3, U_{\beta}=1, L_{\beta}=1$ & $Q_{\alpha}-2 D_{\beta}-L_{\beta}=0$ \\
10 & $Q_{+}=3, Q_{\beta}=4$ & $3 Q_{\beta}-4 Q_{+}=0$ \\
11 & $U_{+}=3, D_{\beta}=-4$ & $3 D_{\beta}+4 U_{+}=0$ \\
\hline \hline
\end{tabular}

Consider a line through this point, $L=\alpha S+\beta R$, for $[\alpha: \beta] \in \mathbb{P}_{\mathbb{Q}}{ }^{1}$, and $R \in \mathbb{P Q}^{n-1}$. Any point in $\mathbf{P Q}^{n-1}$ must lie on such a line, and each line must intersect the surface $C$ at a single other point or be in $C$. Thus by cycling through $R$ (and when appropriate $\alpha$ and $\beta$ ) we can parameterize all solutions to the cubic equation.

For those lines not in $C$ we can find the value of $[\alpha: \beta]$ by substitution into the cubic equation. On such a substitution we obtain

$$
\begin{aligned}
\beta^{2} & \left\{3 \alpha \sum_{i=1}^{n} S_{i}\left[R_{i}^{2}-\left(\sum_{i=1}^{n} R_{i}\right)^{2}\right]\right. \\
& \left.+\beta \sum_{i=1}^{n} R_{i}\left[R_{i}^{2}-\left(\sum_{i=1}^{n} R_{i}\right)^{2}\right]\right\}=0 .
\end{aligned}
$$

Since $\beta=0$ returns $S$ the other point of intersection occurs at

$$
\begin{gathered}
{[\alpha: \beta]=\left[\sum_{i=1}^{n} R_{i}\left\{R_{i}^{2}-\left(\sum_{i=1}^{n} R_{i}\right)^{2}\right\}:\right.} \\
\left.-3 \alpha \sum_{i=1}^{n} S_{i}\left\{R_{i}^{2}-\left(\sum_{i=1}^{n} R_{i}\right)^{2}\right\}\right] .
\end{gathered}
$$

\section{APPENDIX B: CATCHING POINTS ON LINES IN THE QUADRATIC HYPERSURFACE}

In Sec. IV we stated that the parameterization of (15) did not catch all of the solutions: it missed those which sit on lines $L$ through the known solution $\tilde{X}$, which themselves lie in the hypersurface $\Gamma$. Using the construction in Sec. IV with different known solutions $\tilde{X}$, we can ensure that every point can be written as the second intersection of a line $L$ through a point $\tilde{X}$ for which $L \nsubseteq \subset$. Every solution $X$ for which $X^{T} H \tilde{X} \neq 0$ is covered by the known solution $\tilde{X}$ (as $X^{T} H \tilde{X} \neq 0 \Leftrightarrow R^{T} H \tilde{X} \neq 0$ ). Table III lists a set of 11 pairs of $\tilde{X}, X$ for which $X^{T} H \tilde{X}=0$. The only solution $X$ which satisfies all the given conditions is the trivial solution. Thus, any nontrivial solution can be obtained by (15), using an instance of $\tilde{X}$ from Table III.

\section{APPENDIX C: SEMI-FAMILY-UNIVERSAL CASE}

Our choice of $G L(3, \mathbb{Z})$ allows us to study the case where two families have equal charges with relative ease, since we must set $F_{\alpha}$ to zero. This has the effect of reducing the quadratic equation (11) to

$$
\begin{gathered}
2\left\{3\left(Q_{\beta}^{2}-2 U_{\beta}^{2}+D_{\beta}^{2}-L_{\beta}^{2}+E_{\beta}^{2}\right)\right. \\
-4 Q_{+}\left(Q_{\beta}-2 D_{\beta}+3 L_{\beta}+2 E_{\beta}\right) \\
\left.+4 U_{+}\left(2 U_{\beta}+D_{\beta}+E_{\beta}\right)\right\}=0 .
\end{gathered}
$$

We can follow the same procedure as for the FNU case, except now

$$
X^{T} \equiv\left(Q_{+}, U_{+}, Q_{\beta}, U_{\beta}, D_{\beta}, L_{\beta}, E_{\beta}\right)
$$

Suitable choices for $\tilde{X}$ may be read from $5 \leq i \leq 11$ of Table III. 
[1] S. Weinberg, The Quantum Theory of Fields (Cambridge University Press, Cambridge, England, 1995), Vol. 2.

[2] N. Lohitsiri and D. Tong, SciPost Phys. 8, 009 (2020).

[3] J. Davighi, B. Gripaios, and N. Lohitsiri, arXiv:1910.11277.

[4] J. Davighi, B. Gripaios, and N. Lohitsiri (to be published).

[5] P. Fileviez Perez and M. B. Wise, Phys. Rev. D 82, 011901 (2010); 82, 079901(E) (2010).

[6] N. Okada and O. Seto, Phys. Rev. D 82, 023507 (2010).

[7] K. Nakayama, F. Takahashi, and T. T. Yanagida, Phys. Lett. B 699, 360 (2011).

[8] B. Allanach, F. S. Queiroz, A. Strumia, and S. Sun, Phys. Rev. D 93, 055045 (2016); 95, 119902(E) (2017).

[9] N. Okada and S. Okada, Phys. Rev. D 95, 035025 (2017).

[10] N. Okada and S. Okada, Phys. Rev. D 93, 075003 (2016).

[11] N. Okada, S. Okada, and D. Raut, Phys. Lett. B 780, 422 (2018).

[12] P. Agrawal, N. Kitajima, M. Reece, T. Sekiguchi, and F. Takahashi, Phys. Lett. B 801, 135136 (2020).

[13] N. Okada, S. Okada, and D. Raut, Phys. Rev. D 100, 035022 (2019).

[14] J. Heeck and W. Rodejohann, Phys. Rev. D 84, 075007 (2011).

[15] D. Berenstein and E. Perkins, Phys. Rev. D 82, 107701 (2010).

[16] M.-C. Chen, J. Huang, and W. Shepherd, J. High Energy Phys. 11 (2012) 059.

[17] C. D. Carone, L. J. Hall, and H. Murayama, Phys. Rev. D 54, 2328 (1996).

[18] D. E. Kaplan and G. D. Kribs, Phys. Rev. D 61, 075011 (2000).

[19] C. D. Froggatt and H. B. Nielsen, Nucl. Phys. B147, 277 (1979).

[20] R. Gauld, F. Goertz, and U. Haisch, Phys. Rev. D 89, 015005 (2014).

[21] A. J. Buras, F. De Fazio, and J. Girrbach, J. High Energy Phys. 02 (2014) 112.

[22] A. J. Buras and J. Girrbach, J. High Energy Phys. 12 (2013) 009.

[23] W. Altmannshofer, S. Gori, M. Pospelov, and I. Yavin, Phys. Rev. D 89, 095033 (2014).

[24] A. J. Buras, F. De Fazio, and J. Girrbach-Noe, J. High Energy Phys. 08 (2014) 039.

[25] A. Crivellin, G. D’Ambrosio, and J. Heeck, Phys. Rev. Lett. 114, 151801 (2015).

[26] A. Crivellin, G. D’Ambrosio, and J. Heeck, Phys. Rev. D 91, 075006 (2015).

[27] D. Aristizabal Sierra, F. Staub, and A. Vicente, Phys. Rev. D 92, 015001 (2015).

[28] A. Crivellin, L. Hofer, J. Matias, U. Nierste, S. Pokorski, and J. Rosiek, Phys. Rev. D 92, 054013 (2015).

[29] A. Celis, J. Fuentes-Martin, M. Jung, and H. Serodio, Phys. Rev. D 92, 015007 (2015).

[30] A. Greljo, G. Isidori, and D. Marzocca, J. High Energy Phys. 07 (2015) 142.

[31] W. Altmannshofer and I. Yavin, Phys. Rev. D 92, 075022 (2015).

[32] A. Falkowski, M. Nardecchia, and R. Ziegler, J. High Energy Phys. 11 (2015) 173.
[33] C.-W. Chiang, X.-G. He, and G. Valencia, Phys. Rev. D 93, 074003 (2016).

[34] D. Bečirević, O. Sumensari, and R. Zukanovich Funchal, Eur. Phys. J. C 76, 134 (2016).

[35] S. M. Boucenna, A. Celis, J. Fuentes-Martin, A. Vicente, and J. Virto, Phys. Lett. B 760, 214 (2016).

[36] S. M. Boucenna, A. Celis, J. Fuentes-Martin, A. Vicente, and J. Virto, J. High Energy Phys. 12 (2016) 059.

[37] P. Ko, Y. Omura, Y. Shigekami, and C. Yu, Phys. Rev. D 95, 115040 (2017).

[38] R. Alonso, P. Cox, C. Han, and T. T. Yanagida, Phys. Rev. D 96, 071701 (2017).

[39] R. Alonso, P. Cox, C. Han, and T. T. Yanagida, Phys. Lett. B 774, 643 (2017).

[40] Y. Tang and Y.-L. Wu, Chin. Phys. C 42, 033104 (2018).

[41] C.-H. Chen and T. Nomura, Phys. Lett. B 777, 420 (2018).

[42] G. Faisel and J. Tandean, J. High Energy Phys. 02 (2018) 074.

[43] K. Fuyuto, H.-L. Li, and J.-H. Yu, Phys. Rev. D 97, 115003 (2018).

[44] L. Bian, H. M. Lee, and C. B. Park, Eur. Phys. J. C 78, 306 (2018).

[45] M. Abdullah, M. Dalchenko, B. Dutta, R. Eusebi, P. Huang, T. Kamon, D. Rathjens, and A. Thompson, Phys. Rev. D 97, 075035 (2018).

[46] D. Bhatia, S. Chakraborty, and A. Dighe, J. High Energy Phys. 03 (2017) 117.

[47] B. C. Allanach, B. Gripaios, and T. You, J. High Energy Phys. 03 (2018) 021.

[48] B. C. Allanach, T. Corbett, M. J. Dolan, and T. You, J. High Energy Phys. 03 (2019) 137.

[49] B. Allanach and J. Davighi, J. High Energy Phys. 12 (2018) 075 .

[50] G. H. Duan, X. Fan, M. Frank, C. Han, and J. M. Yang, Phys. Lett. B 789, 54 (2019).

[51] C.-Q. Geng and H. Okada, arXiv:1812.07918.

[52] J. Kawamura, S. Raby, and A. Trautner, Phys. Rev. D 100, 055030 (2019).

[53] B. C. Allanach, J. M. Butterworth, and T. Corbett, J. High Energy Phys. 08 (2019) 106.

[54] S. Dwivedi, A. Falkowski, D. K. Ghosh, and N. Ghosh, arXiv:1908.03031.

[55] B. C. Allanach and J. Davighi, Eur. Phys. J. C 79, 908 (2019).

[56] W. Altmannshofer, J. Davighi, and M. Nardecchia, Phys. Rev. D 101, 015004 (2020).

[57] L. Calibbi, A. Crivellin, F. Kirk, C. A. Manzari, and L. Vernazza, arXiv:1910.00014.

[58] R. Aaij et al. (LHCb Collaboration), Phys. Rev. Lett. 113, 151601 (2014).

[59] R. Aaij et al. (LHCb Collaboration), J. High Energy Phys. 08 (2017) 055.

[60] G. Hiller and F. Kruger, Phys. Rev. D 69, 074020 (2004).

[61] B. C. Allanach, J. Davighi, and S. Melville, J. High Energy Phys. 02 (2019) 082.

[62] D. B. Costa, B. A. Dobrescu, and P. J. Fox, Phys. Rev. Lett. 123, 151601 (2019).

[63] B. C. Allanach, B. Gripaios, and J. Tooby-Smith, arXiv: 1912.04804. 